https://helda.helsinki.fi

\title{
The software and hardware architecture of the real-time protection of in-vessel components in JET-ILW
}

\section{JET Contributors}

2019-07

JET Contributors, Hatch , D R , Ahlgren , T , Aho-Mantila , L , Airila , M , Björkas , C , Heinola , K, Lahtinen, A, Nordlund, K, Safi , E, Pehkonen, S-P , Salmi , A, Tala , T , Huber , V \& Jarvinen , A 2019 , ' The software and hardware architecture of the real-time protection of in-vessel components in JET-ILW ' , Nuclear Fusion , vol. 59 , no. 7 , 076016 . https://doi.org/10.1088/1

http://hdl.handle.net/10138/326697

https://doi.org/10.1088/1741-4326/ab1a79

unspecified

acceptedVersion

Downloaded from Helda, University of Helsinki institutional repository.

This is an electronic reprint of the original article.

This reprint may differ from the original in pagination and typographic detail.

Please cite the original version. 


\title{
The Software and Hardware Architecture of the Real-Time Protection of In-Vessel Components in JET-ILW
}

\author{
V. Huber ${ }^{1}$, A. Huber ${ }^{2}$, D. Kinna ${ }^{3}$, G. Sergienko², I. Balboa ${ }^{3}$, S. Brezinsek ${ }^{2}$, P. McCullen ${ }^{3}$,
} P. J. Lomas ${ }^{3}$, G. F. Matthews ${ }^{3}$, P. Mertens ${ }^{2}$, F. G. Rimini ${ }^{3}$, and K.-D. Zastrow ${ }^{3}$

\author{
The JET Contributors \\ ${ }^{1}$ Supercomputing Centre, Forschungszentrum Jülich, Jülich, Germany \\ ${ }^{2}$ Institute of Energy and Climate Research, Forschungszentrum Jülich, Jülich, Germany \\ ${ }^{3}$ Culham Centre for Fusion Energy (CCFE), Culham Science Centre, Abingdon, UK
}

Corresponding Author: V. Huber, valentina.huber@ukaea.uk

The JET ITER-like wall (JET-ILW) combines plasma-facing components (PFC) made of bulk beryllium for main chamber limiter tiles and of bulk tungsten as well as tungsten coated CFC tiles for divertor tiles. The risk of damaging the metallic PFCs caused by beryllium melting or cracking of tungsten owing to thermal fatigue required a new reliable active protection system. To address this issue, a real-time protection system comprising newly installed imaging diagnostics, real time algorithms for hot spot detection and alarm handling strategy has been integrated into the JET protection system.

This contribution describes the design, implementation, and operation of the near infrared (NIR) imaging diagnostic system of the JET-ILW plasma experiment and its integration into the existing JET protection architecture. The imaging system comprises four wide-angle views, four tangential divertor views, and two top views of the divertor. Regions of interest (ROI) on the selected PFCs of different materials are analyzed in real-time and the maximum temperature measured in each ROI is sent to a real-time algorithm called vessel thermal map (VTM) to determine the likely cause of the overheating and to request an appropriate response from the plasma control system. Postpulse data visualization and advance analysis of all types of imaging data is provided by the new software framework JUVIL (JET Users Video Imaging Library). The hot spots formation at the reionization zones due to impact of the reionized neutrals as well as due to RF-induced fast ion losses is recognized as a big threat due to quick surface temperature rise. Because it could trigger the protection system to stop a pulse, it is important to identify the mechanisms and conditions responsible for the formation of such hot spots. To address this issue a new software tool Hotspot Editor has been developed.

Future development of the JET real-time first wall protection is focussed on the DT campaign and the ITER relevant conditions which will cause failure of camera electronics within the Torus hall. To provide the reliable wall protection, two more sensitive logarithmic NIR camera systems equipped with new optical relays to take images and cameras outside of the biological shield have been installed on JET-ILW and calibrated with in-vessel calibration light source. 


\title{
THE SOFTWARE AND HARDWARE ARCHITECTURE OF THE REAL-TIME PROTECTION OF IN-VESSEL COMPONENTS IN JET-ILW
}

\author{
V. HUBER ${ }^{1}$, A. HUBER ${ }^{2}$, D. KINNA ${ }^{3}$, G.F. MATTHEWS ${ }^{3}$, G. SERGIENKO ${ }^{2}$, I. BALBOA ${ }^{3}$, S. BREZINSEK ${ }^{2}$, \\ P. J. LOMAS ${ }^{3}$, J. MAILLOUX ${ }^{3}$, P. MCCULLEN ${ }^{3}$, PH. MERTENS ${ }^{2}$, F. G. RIMINI ${ }^{3}$, K-D. ZASTROW ${ }^{3}$ and JET \\ CONTRIBUTERS*
}

Email: $\underline{\text { V.Huber@fz-juelich.de }}$

[1] Forschungszentrum Jülich GmbH, Supercomputing Centre, Jülich, Germany

[2] Forschungszentrum Jülich GmbH, Institut für Energie- und Klimaforschung - Plasmaphysik, Jülich, Germany

[3] CCFE, Culham Science Centre, Abingdon, UK

[*] See the author list of "X. Litaudon et al 2017 Nucl. Fusion 57 102001"

\begin{abstract}
The JET ITER-like wall (JET-ILW) combines plasma-facing components (PFC) made of bulk beryllium for main chamber limiter tiles and of bulk tungsten as well as tungsten coated CFC tiles for divertor tiles. The risk of damaging the metallic PFCs caused by beryllium melting or cracking of tungsten owing to thermal fatigue required a new reliable active protection system. To address this issue, a real time protection system comprising newly installed imaging diagnostics, real time algorithms for hot spot detection and alarm handling strategy has been integrated into the JET protection system.

This contribution describes the design, implementation, and operation of the near infrared (NIR) imaging diagnostic system of the JET-ILW plasma experiment and its integration into the existing JET protection architecture. The imaging system comprises four wide-angle views, four tangential divertor views, and two top views of the divertor. Regions of interest (ROI) on the selected PFCs of different materials are analysed in real time and the maximum temperature measured in each ROI is sent to a real time system known as the vessel thermal map (VTM) to determine the likely cause of the overheating and to request an appropriate response from the plasma control system. Post-pulse data visualization and advance analysis of all types of imaging data is provided by the new software framework JUVIL (JET Users Video Imaging Library). The formation of hot spots is recognized as a significant threat due to rapid surface temperature rise. Because it could trigger the protection system to stop a pulse, it is important to identify the mechanisms and conditions responsible for the formation of such hot spots. To address this issue new software tools Hotspot Editor and Viewer have been developed. Future development of the JET real time first wall protection is focused on the D-T campaign and the ITER relevant conditions which will cause failure of camera electronics within the Torus hall. To provide the reliable wall protection, two more sensitive logarithmic NIR camera systems equipped with new optical relays to take images and cameras outside of the biological shield have been installed on JET-ILW and calibrated with an in-vessel calibration light source.
\end{abstract}

\section{INTRODUCTION}

The JET ITER-like wall (JET-ILW) combines plasma-facing components (PFC) made of bulk beryllium for main chamber limiter tiles and of bulk tungsten as well as tungsten coated CFC tiles for divertor tiles [1]. The risk of damaging the metallic PFCs caused by beryllium melting or cracking of tungsten owing to thermal fatigue required a new reliable active protection system to avoid damage of the plasma-facing components. To address this issue, a real time protection system comprising newly installed imaging diagnostics, real time algorithms for hot spot detection and alarm handling strategy has been implemented into the JET protection system. Considering the different material properties of ILW-PFCs the real time imaging system must fulfil several objectives such as avoiding the melting of the Be-tiles, minimizing the risk of delamination of the tungsten coated tiles and keeping the surface temperature below the threshold at which bulk tungsten re-crystallizes $\left(1200^{\circ} \mathrm{C}\right)[2]$. This means that the system which monitors the surface temperature of the PFCs must be active in every plasma discharge. Such a sophisticated protection becomes especially important during the preparation and execution of the experiments of 
the coming D-T campaign, where stationary plasmas with additional power of $40 \mathrm{MW} / 5 \mathrm{~s}$ and with tolerable wall heat loads and impurity concentration are required.

This contribution describes the design, implementation, and operation of the near infrared imaging diagnostic system of the JET-ILW plasma experiment and its integration into the existing JET protection hardware and software architecture.

\section{NEAR INFRARED IMAGING DIAGNOSTIC SYSTEMS}

The JET-ILW imaging system for machine protection [3] is based on analogue monochrome CCD cameras (Hitachi KP-M1AP; sensor: Sony ICX423AL, Sensor size 768x576, pixel size: $11.6 \mu \mathrm{m}(\mathrm{H}) \times 11.2 \mu \mathrm{m}(\mathrm{V})$ ), equipped with Near InfraRed (NIR) filters operating in the near infrared spectral region (970nm-1300nm). Synchronized with the external sync signals (V/H scans), the cameras operate in non-interlaced mode at 50 fields per second with binning (odd and even lines are exposed together at the same time). In this mode, the spatial resolution is lost in the vertical direction: the camera delivers images with an apparent size of $720 \times 288$ pixels every $20 \mathrm{~ms}$. The pixels are effectively larger in the vertical direction, and hence more sensitive. The imaging system comprises four wide-angle views, four tangential divertor views, and two top views of the divertor. The cameras are used to measure the surface temperature of the PFCs with an exposure time of 20ms. The operation temperature ranges of NIR protection cameras for the materials used on JET are: $\mathrm{Be} 700-1400^{\circ} \mathrm{C}$; W coating 700 $1370^{\circ} \mathrm{C} ; \mathrm{W} 700-1400^{\circ} \mathrm{C}$.

\section{VIDEO DIGITIZATION AND DISTRIBUTION}

A data flow diagram which includes fundamental camera hardware and data processing components is shown in Fig.1. The analogue composite video signal from each CCD camera is digitized by a Gigabit Ethernet (GigE) frame grabber iPORT PT1000-ANL (2-6-V2-E). The frame grabber shares the digital video data across a dedicated network to three separate PCs simultaneously: the camera Real Time Processing system, a Video Capture system for data storage, and the Live Display. This setup is replicated for each Hitachi camera. The new 'Widy' logarithmic response cameras (see Section 10) currently in commissioning generate digital data directly via USB. For these cameras a single PC manages the real time processing, capture and propagates data for live display, using separate processes in a logical structure that mimics the architecture above.

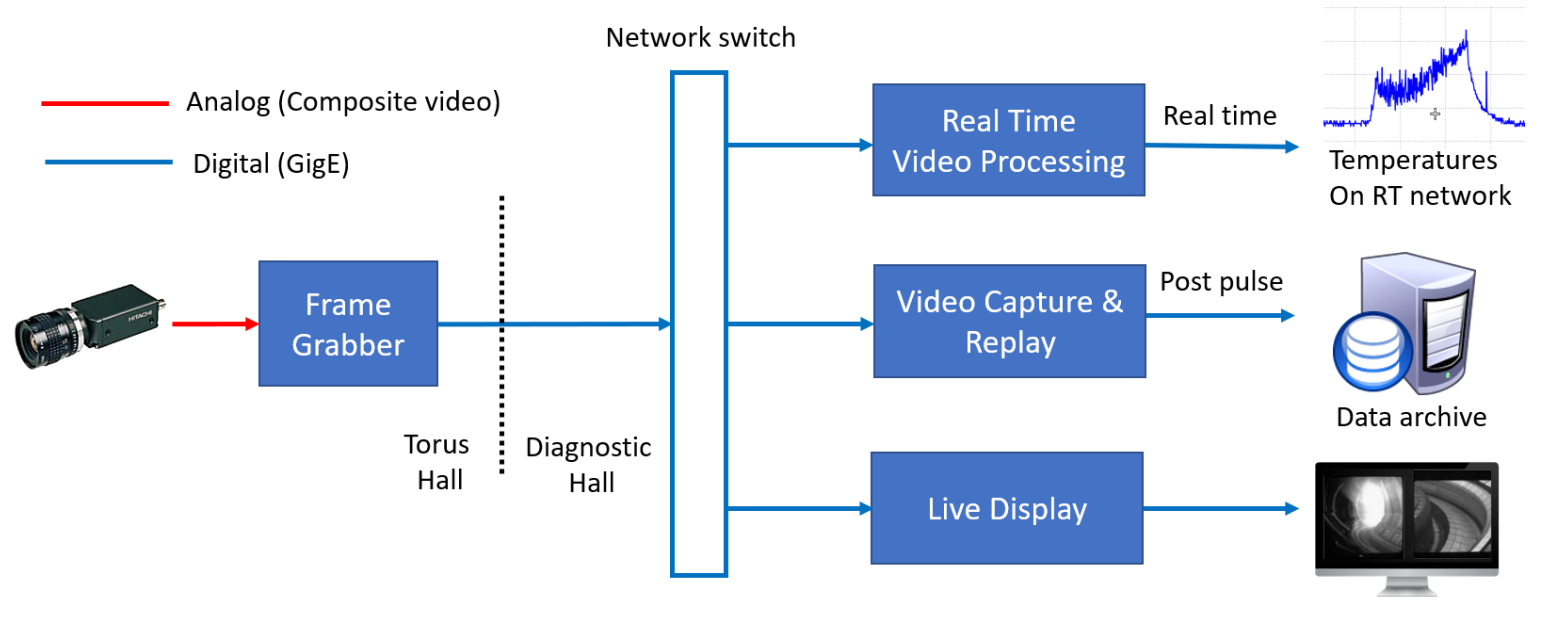

\section{REAL TIME PROTECTION}

The cameras act as temperature sensors. A real time processing system on each camera converts pixel intensities to the surface temperature. This system monitors Regions Of Interest (ROIs) on the selected PFCs of different materials, and outputs the maximum temperature measured in each ROI to a separate real time system known as the Vessel Thermal Map (VTM) [4]. If the temperature from an active ROI of some camera crosses a threshold for a vessel wall segment, the VTM classifies this as a 'Main chamber Hot Spot' or a 'Divertor Hot Spot' depending on where the event happens and triggers an alarm. It is the VTM that communicates with the Real Time Protection Sequencer (RTPS), which in turn decides how the control actuators should respond. The response of the RTPS is programmable and takes into account many inputs in addition to those from imaging diagnostics. The action is usually not a hard, fast, stop, but instead a pre-programmed controlled plasma termination, called 'soft landing,' to reduce the risk of a disruption. The algorithm for real time image processing and elimination of pixels 
which should not contribute to the calculation because they are caused by sensor aberrations or transient phenomena such as neutron hits, and its implementation, are described in [3]. The overall system, from the ROIs to the responses, is highly configurable. The tool that does such configuration at JET and ensures that it is both consistent and audited is called 'Level 1'.

\section{POST-PULSE DATA VISUALIZATION AND ADVANCED ANALYSIS}

\subsection{JUVIL VIDEO IMAGING SOFTWARE}

The JUVIL framework consists of modular object-oriented components written in Python [5]. They provide components and functions for loading and post-processing of video data, which are highly configurable and can be easily extended and adapted for new imaging cameras, data formats or developing applications for users' own analysis. There is a separation between generic interfaces and site-specific implementations, e.g. JET specifics are implemented in the jet package. The object-oriented hierarchical structure provides a very efficient common platform with the possibility to reuse the functionality of existing components without the need to copy the code several times and makes the software maintenance much easier. For example, the VVideo component provides generic properties and functions to access video data and JVideo is a subclass of VVideo which implements these functions using getdat library to access JET data. The PIWVideo is a subclass of JVideo and it includes additional features and functions for protection cameras.

\subsection{JUVIL GRAPHICAL INTERFACE}

Fig. 2 shows JUVIL graphical interface for post-processing of videos from various imaging systems [5]. It displays the list of JET cameras grouped into categories: Protection for protection NIR cameras, Operation for CCD colour cameras, Experiment for scientific IR and VIS cameras, Intershot for post-pulse videos, Tomography for tomographic reconstructions and Other for all other types. To improve the performance the data sizes are refreshed only for those groups which are expanded, and the videos are loaded in chunks of 100 frames without waiting
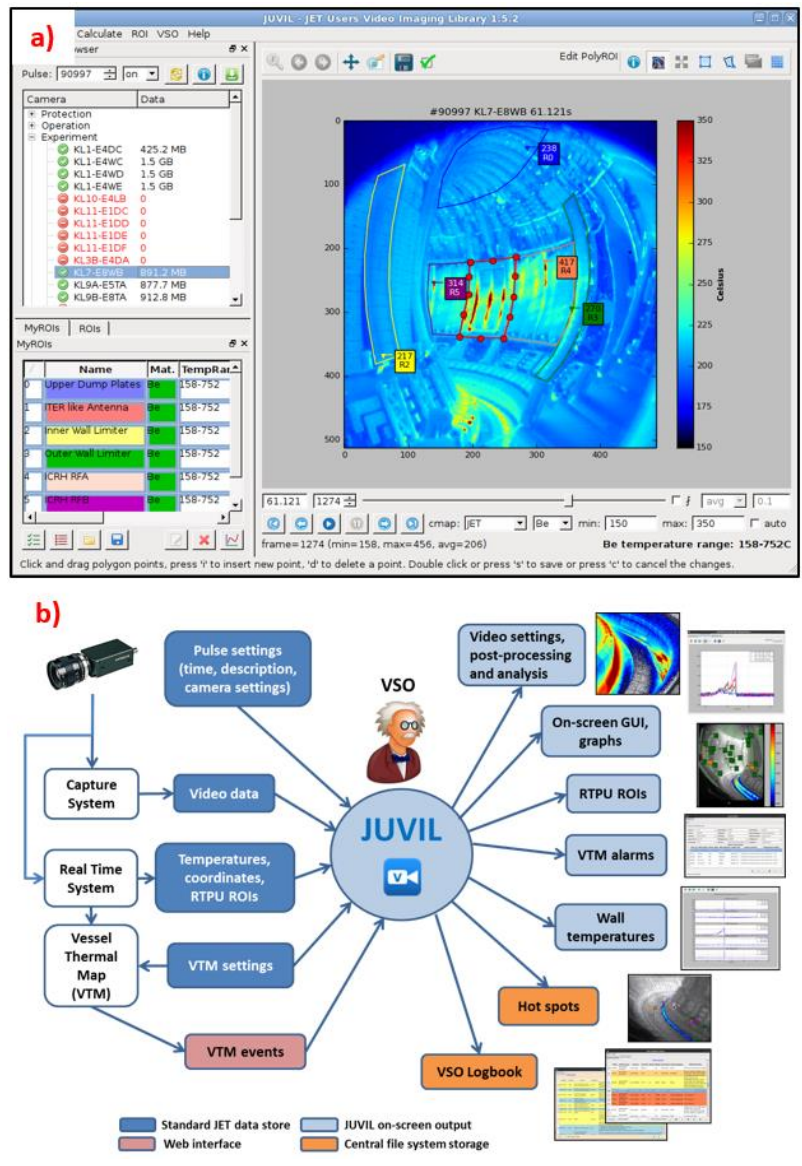

FIG.2. a) JUVIL graphical Interface, b) VSO Tools until the whole video is loaded. The initial time is synchronized to the time of the previously loaded video to compare the images from several cameras. JUVIL automatically carries out the video post-processing and shows to a user the information about video settings. It also calibrates a digital level count of each video pixel into the temperature using the real time processing algorithm: subtracts the offset, performs dead pixels and flat field corrections, and maps the results to corresponding temperatures of materials used at JET. If the video offset is not available, JUVIL calculates it as the averaged frame of the first 10 video frames. It assumes thereby that the temperatures at the beginning of the pulse are not too hot. Furthermore, the user can select the temperature range to be displayed. The temperatures below a specified minimum are hidden, and it enables to overlay the video with the camera image to see the distribution of hot regions inside the vessel. The ROIs panel contains the list of ROIs which are monitored by a real time processing system on each camera and shows their locations and temperatures. In addition, a user could specify custom ROIs, modify their attributes, shapes and locations, and save the ROIs to the file system to use them later. Furthermore, JUVIL calculates maximum, average, and standard deviation profiles. 


\subsection{VSO TOOLS}

The primary role of the viewing system operator (VSO) is to assist the session leader, engineer in charge and scientific coordinator in the interpretation of an alarm sent by the VTM due to a protection camera during experiments. To simplify the job of the VSO, some new features, the so-called VSO, were integrated into JUVIL for the interpretation of VTM events and for the quick post-pulse analysis of video data required for the preparation of the next plasma pulse [5]. They enable to load automatically the list of VTM alarms raised for a specific pulse, plot the temperatures of wall segments with the corresponding alarm thresholds and store categorised alarms into the VSO logbook tools (see Fig. 2b). This saves a significant amount of time, which can be used by the VSO for actual analysis of the event.

\subsection{TOOLS FOR THE STUDY OF HOT SPOTS}

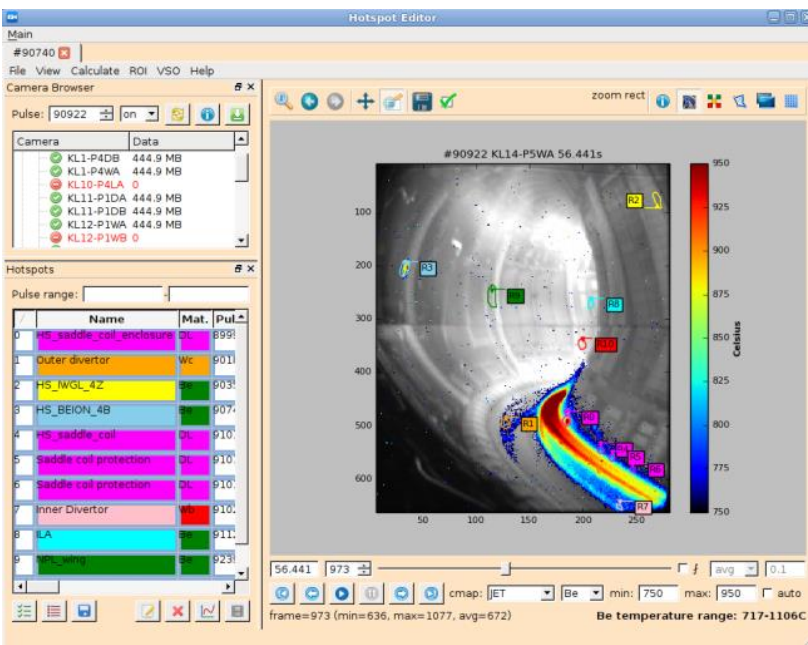

FIG.3. Hotspot Editor

The hot spots are localized micro-regions on the surface that appear to have much higher temperatures than the surrounding regions. Because the hot spots raise VTM alarms, which can cause the protection system to stop a pulse where it may not be necessary, it is important to study the appearances of new hot spots, their locations and temperatures as well as the conditions of disappearance of the existing hot spots. For this aim, the new Hotspot Editor and Viewer, were integrated into JUVIL [6]. The Hotspot Editor automatically loads the hot spots for a specific camera stored in the catalogue (see Fig.3). The attributes of hot spots are similar to ROIs, but they contain in addition the time when a hot spot was detected or modified, the measured temperature, the flag whether a hot spot caused a VTM alarm, and optionally the time when a hot spot clearly disappeared (e.g. after the cleaning up or the replacement of tiles). It is possible to load automatically the corresponding videos from times when a hot spot was detected or modified as well as to plot its temperatures. The Hotspot Viewer displays the catalogue of all detected hot spots and shows their evolution. It provides also the filter options for extraction of hot spots during a pulse range.

\section{HOT SPOT ON THE INNER WALL GUARD LIMITER}

The Hotspot Editor is designed for the analysis of hot spots with temperatures below as well beyond the trip level. It calculates the history of temperatures of hot spots using a thresholding technique to study hot spot temperature evolution. Fig. 4a shows an example of a hot spot in the main chamber of JET. The hot spot is located at the upper part of the inner wall guard limiter (IWGL). Thanks to the Hotspot Viewer, the evolution of this specific hot spot could be followed (see Fig.4b). The first time IWGL hot spot was observed during an ohmic discharge at pulse number 87643 . In this pulse the surface temperature of Be tile reaches the trip level of $925^{\circ} \mathrm{C}$ and remains above this trip level for time window shorter than the assertion time, which is 200ms. Correspondingly, the alarm was not triggered during this pulse. During the following pulses (e.g. \#87728), the surface temperature remains beyond the trip level longer than the assertion time (see Fig.4c). As a result, the trip alarm is triggered and VTM sends an alarm to RTPS
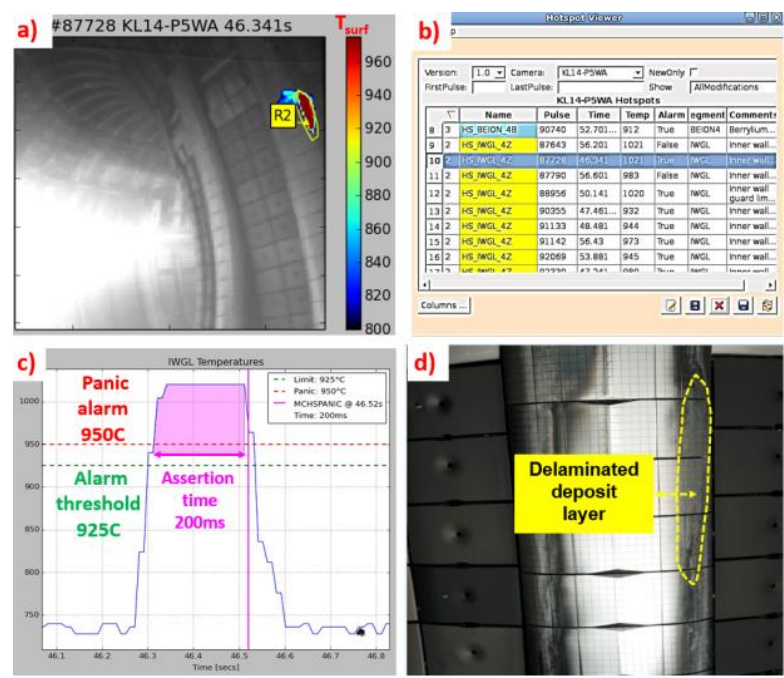

FIG.4. a) Hot spot on the inner wall guard limiter (IWGL); b) Hotspot Viewer catalogue of detected hot spots; c) Surface temperature of the IWGL hot spot; d) High-resolution image of the inner wall guard limiter. 
requesting a recovery action from the plasma control systems. The action of the real-time protection system during this hot spot formation is described in detail in [6]. The surface temperature measured by the protection system shows a fast initial rise which suggests that the measurement is not the result of thermal radiation from the bulk. Such a temporal response of the temperature (quick increase and decrease) is the typical signature for a deposited layer with poor thermal contact with the material substrate. The analysis shows also the slight growth of the size of IWGL spot. It could be explained by the progressing delamination of the deposited layer from the bulk material substrate. That was confirmed by the high-resolution images taken during the vessel survey (see Fig.4d).

\section{HOT SPOT ON THE ITER-LIKE ANTENNA}

The hot spot editor is designed for the analysis of the hot spots with temperatures below as well as beyond the trip level. It calculates temperatures of hot spots for an arbitrary pulse as well as enables to plot or store the history of temperatures for specific pulses or pulse range using a thresholding technique to study hot spot temperature evolution. Fig 5 shows the hot spot behaviour on the ITER like antenna (ILA) in a pulse range spanning over 5000 pulses. The ILA is one of the additional heating systems at JET which was designed to couple 7.2MW $\left(8 \mathrm{MW} / \mathrm{m}^{2}\right)$ across the frequency range $30-55 \mathrm{MHz}$ [8]. Hot spots are the potentially most dangerous events related to ILA operation. If left uncontrolled they can produce damage and cause plasma disruption by impurity influx. The surface temperature threshold was set for this analysis to $950^{\circ} \mathrm{C}$ and the assertion time to $120 \mathrm{~ms}$. To exclude the hot spots during disruptions, these criteria were crosschecked with the disruption database which contains the full information about disruptions: pulse, time, etc. Fig. 5c demonstrates the formation of 10 hot spots on the antenna grids and how they correlate with plasma shape

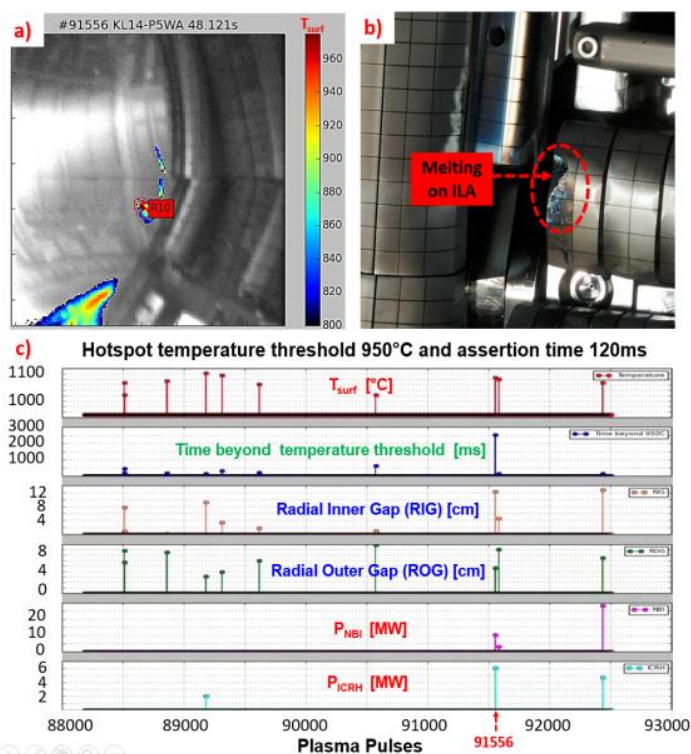

FIG.5. Analysis of the hot spot on the ITER like antenna in a pulse range from 88000 to 92504.

ROGs) which define the distance between the inner and outer limiter, respectively, and the plasma. Furthermore, it shows the values of the additional heating power by NBIs and ICRH heating systems. The hot spots around the pulse 8900 were formed in the pulses with small RIG and large ROG values. Additionally, the temperatures remained beyond the temperature threshold for the relatively short times: below 300ms. These are indicators that these hot spots have causes which are not related to the overheating of the antenna components. Analysis shows that these hot spots were triggered by the bremsstrahlung during the wall MARFE formation at the end of the plasma discharge during the disruption phases. They are clearly "false hot spots". This knowledge was used to deselect such hot spots from the data base. The genuine hot spot at pulse 91556 is related to the overheating of the antenna corner tile of Faraday screen.

\section{HOT SPOTS ON THE PARTLY DELAMINATED DIVERTOR TILES}

Delamination of tungsten coatings on a few CFC based tiles was observed after the campaigns in 2013-2014 [5]. Significant heat loads in this region have resulted in a pattern of film cracking and delamination along CFC fibre planes in a toroidal orientation along the tile (Fig.9b). There is a known mechanism for failure of the coatings by carbidisation if surface temperatures exceed $1350^{\circ} \mathrm{C}$ for more than two hours [10]. However, this mechanism has not been identified in the samples analysed so far because the protection limit is below this temperature. Delamination along fibre planes is also created by a fatigue due to the mismatch in thermal expansion and is intrinsically variable due to complex 3D structure of CFC materials. Fig. 5a shows the mapping of hot spots detected on the divertor. Most of these hot spots form during the ELMy H-mode plasma discharge $\left(\mathrm{B}_{\mathrm{T}} \approx 2.6 \mathrm{~T}\right.$, $\mathrm{Ip}=2.5 \mathrm{MA}$ ) with the additional input power of about 18.0 MW in low-triangularity magnetic equilibria with the strike points located on the vertical targets. The temperature of the hot spots rises quickly during the ELM and cools down abruptly when the ELM is over (Fig.5c). This is a typical behaviour of overheated edges of delaminated layers. In the case shown, the ELM frequency is about $15 \mathrm{~Hz}$. At such a frequency the hot spots are seen on every third image frame. The temperature of the hotspots remains below the trip level (here $1105^{\circ} \mathrm{C}$ ) during the entire pulse and therefore does not trigger the VTM alarm. Even if the temperature of the hot spots would have exceeded the trip level, at such ELM frequencies the time duration when the surface temperature is 

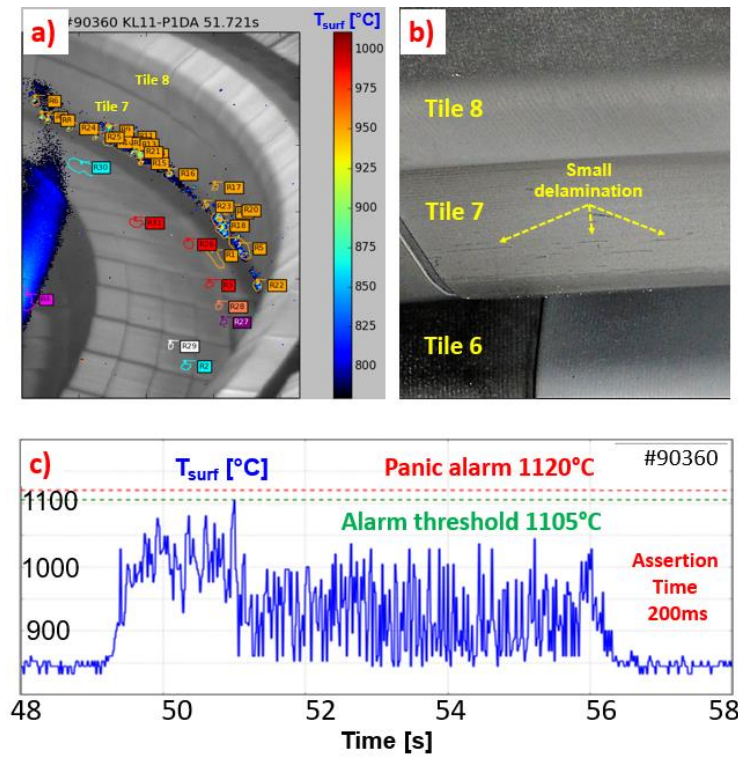

FIG.6. a) Hot spots on the lower outer vertical target (Tile7) during the ELMy H-mode plasma discharge; $b$ ) Delamination of $W$ coatings; $c$ ) Measured surface temperature profile of Tile 7. above the trip level (in this case 20ms) is shorter than the assertion time, hence the transient temperature rise would not have triggered an alarm. The NIR cameras used for protection [3] operate in non-interlaced mode at 50 fields per second. ELMs with frequency below $25 \mathrm{~Hz}$ will lead to transient hot spot formation during alternate frame images and, correspondingly, will be ignored by the protection system. The strength of ELMs can be deduced from the loss in stored plasma energy. With an increase of the ELM frequency the loss in stored plasma energy during the ELM, the ELM energy loss, decreases because the ELM size is inversely proportional to the ELM frequency [10]. For JET-ILW plasmas with an available auxiliary heating power of about 28MW and the heating power duration according the JET-ILW requirements, the ELMs with frequency beyond $25 \mathrm{~Hz}$ do not lead to an increase of the temperature to values above the trip level.

\section{STATISTIC OF VTM ALARMS}

All VTM alarms on the JET-ILW machine are well characterised and catalogued since the C31 campaign when the Real-Time Protection system started to operate routinely. Fig. 7 shows the number of alarms per bin of 500 plasma discharges. The genuine alarms include the genuine hot spots as well as classical heat load events which demonstrate a classical heat up and cool down behaviour: a slow increase of the temperature by applying heat loads as well as a slow temperature reduction after removal of the heat load source. A large number of VTM alarms were triggered during the first 3 campaigns, C31, C32 and C33. Majority of these alarms was due to classical heat up of the first wall components, due to hot spots formation and due to heating of the NBI beam reionization zones (see Fig.7b and 7c). The reionization zones are the areas on the outer limiters which are subjected to the impact of the re-ionised neutrals injected by the heating system. The classical heat up of the wall components occurred mainly during the H-mode plasmas with additional neutral beam as well as ICRH heating. In the early pulses, the predominant location of the alarms in the divertor region was the divertor tile 5 (horizontal target), while later, the number of alarms from tile 6 signals increased significantly. Around the 86501 and 87000 pulse marks, an increased alarm numbers were noted, mostly triggered by temperature signals from divertor tiles 5, 6 and 7. The maximal number of alarms of about 70 was triggered at mark 87001 which correspond to the pulse range of 87001-87500. During this pulse range about $14 \%$ of plasma discharges were terminated to avoid harmful situations like dangerous overheating through classical heat up of the wall components. Averaged over the entire C33 campaign, about $8 \%$ of the plasma discharges were terminated by RTPS. Also, the number of alarms due the NBI beam re-ionization during the $\mathrm{C} 33$ campaign reached the maximum of $2-3 \%$.

The following campaigns C34-C36 show a general tendency of reduction of the VTM alarms because of the continuous improvement of the real-time protection system as well as a better understanding of the physics of events leading to the alarms. Within the last experimental campaigns C37 and C36b a significant improvement of the auxiliary heating systems on JET-ILW 
has been performed leading to a marginal increase of the plasma terminations due to the RTPS safety system: about $2-3 \%$ are due to the classical heat up and about $1-1.5 \%$, to the hot spot formation. As a result, the false alarms were reduced to less than $0.5 \%$ of all plasma discharges, even though the total additional heating power increased significantly in these campaigns.

\section{FUTURE DEVELOPMENT OF THE JET REAL TIME FIRST WALL PROTECTION}

The future development of the JET real time first wall protection is focused on the D-T campaign and on operation near ITER relevant conditions. D-T operation at JET will cause failure of camera electronics within the Torus hall due to significant increase of the hard radiation level (neutrons/gammas). To provide the reliable wall protection needed during the coming D-T campaign, two camera systems, equipped with new optical relays to take the images and the cameras outside of the biological shield, have been installed on JET-ILW and calibrated with an in-vessel calibration light source $[12,13]$. The optical design concept of both systems with wide angle view as well as the divertor view is based on reflective optics, mainly to be able to sustain high neutron radiation. Similar to the ITER conditions, it transports the light by reflective optics (mirror systems) over long distances (>40 m) to the detection system located outside the biological shield. Figures 8 and 9 show the mechanical layout and fields of view of the wide-angle and of the divertor imaging systems, respectively. The location of the cameras outside the biological shield makes it possible to integrate more sensitive and sophisticated cameras in the wall protection system.
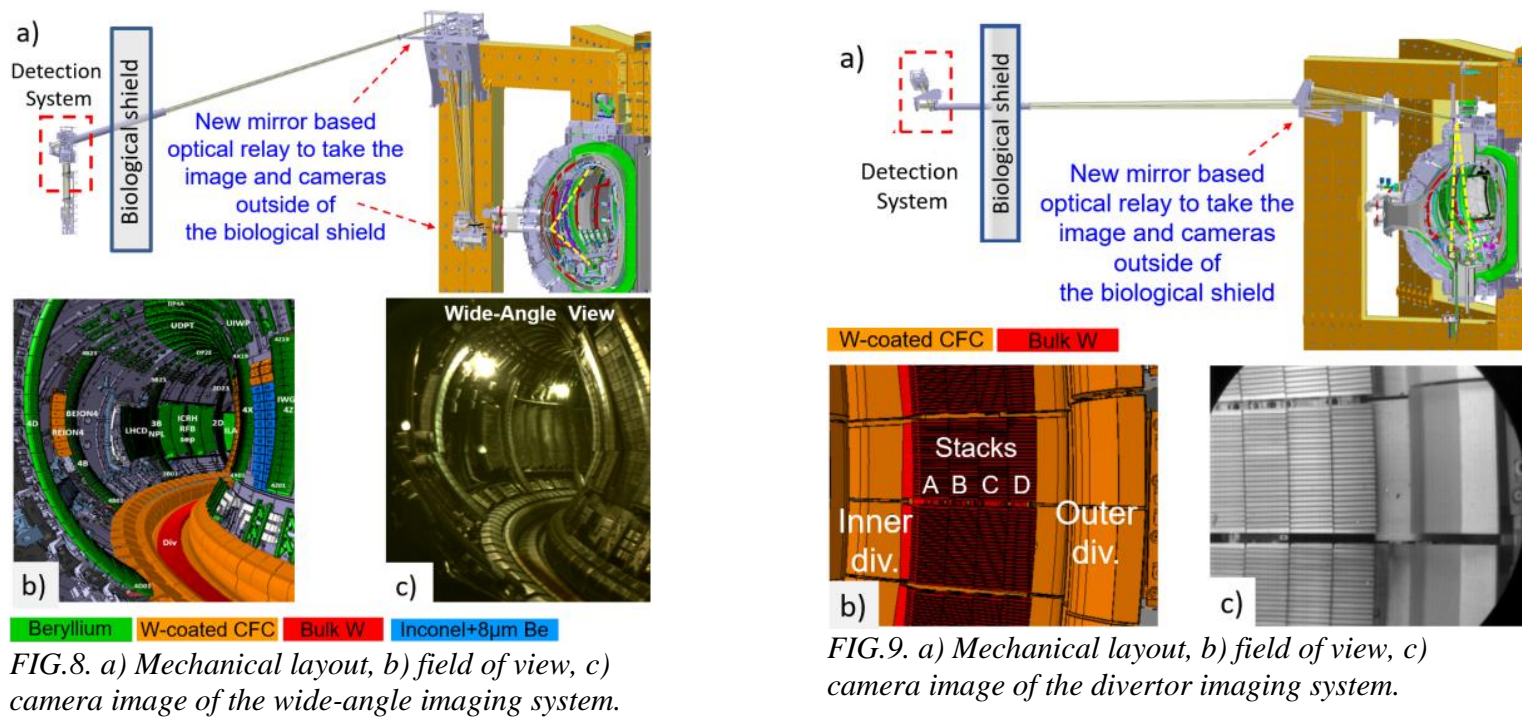

FIG.9. a) Mechanical layout, b) field of view, c) camera image of the divertor imaging system.

The new JET imaging system for machine protection is equipped now with new digital logarithmic NIR cameras: New Imaging Technologies (NIT), Widy SWIR 640U-ST and 640V-ST cameras, resolution 640×512 pixel, pixel size $15 \mu \mathrm{m} \times 15 \mu \mathrm{m}$, max. $200 \mathrm{fps}$, dynamic range 14bits, USB2 or USB3 data connection interface, logarithmic response [14]. These NIR-SWIR cameras use InGaAs (Indium Gallium Arsenide) detectors, offering a high quantum efficiency (QE>70\%) at the wavelength range 900-1700nm. For these cameras, a band pass filter $1200 \mathrm{~nm} \pm 10 \mathrm{~nm}$ is used. The choice of the camera and the central wavelength of the filter is based on the analysis reported in [3]. There are advantages of the usage of the optimised wavelength $(\lambda=1.2 \mu \mathrm{m})$ for the surface temperature measurements: temperature independent spectral emissivity for tungsten; less sensitivity of the measurements to the surface roughness; reduced maximum relative error. All camera sensors are cooled to temperatures of about $0^{\circ} \mathrm{C}$ to reduce the dark noise which is less at lower temperatures. One example of the calibration of the wide angle Widy camera by ICLS is shown in Fig.10a: digital levels measured by the camera versus brightness temperatures of the ICLS with one lamp and with a combination of two lamps. The Planck radiation curve was used as a fit of the experimental data. The

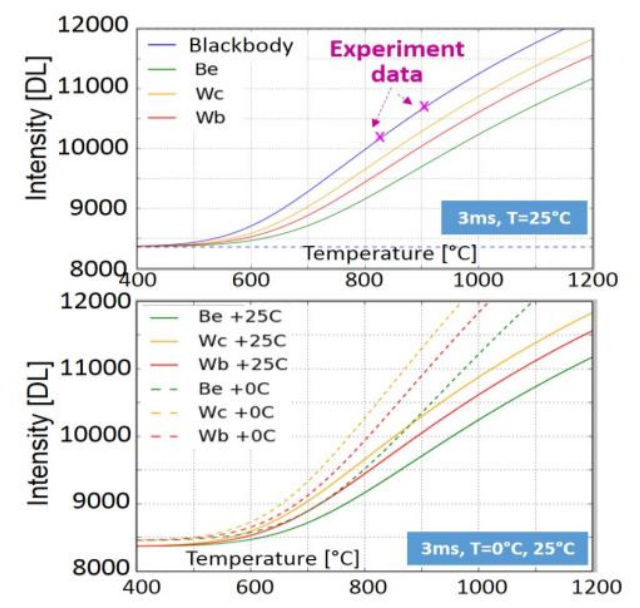

FIG.10. a) Digital levels measured by the camera versus brightness temperatures of the ICLS with one and two lamps b) Calibration for sensor temperatures: $0^{\circ} \mathrm{C}$ and $25^{\circ} \mathrm{C}$. 
calibration curve could be easily adapted to the wanted material as shown in Fig.10a: yellow for W coated, red for bulk tungsten and green for beryllium. During the calibration, it was recognised that the selected InGaAs sensor is sensitive to changes of sensor temperatures as shown in Fig.10b. For the sensor temperature of $0^{\circ} \mathrm{C}$ the camera demonstrates a higher sensitivity. Consequently, cameras were calibrated for several sensor temperatures and times exposures which will be used during the experiments. These developments and improvements mean that the JET Real-time protection from intolerable temperatures will remain effective during DTE2.

\section{ACKNOWLEDGEMENTS}

This work has been carried out within the framework of the EUROfusion Consortium and has received funding from the Euratom research and training programme 2014-2018 under grant agreement No 633053. The views and opinions expressed herein do not necessarily reflect those of the European Commission.

\section{REFERENCES}

[1] MATTHEWS, G. F. et al, "JET ITER-like wall - overview and experimental programme”, 2011 Phys. Scr.T145 014001.

[2] MERTENS, PH et al, "Clamping of solid tungsten components for the bulk W divertor row in JET - precautionary design for a brittle material”, 2009 Phys. Scr. T138 014032.

[3] HUBER, A. et al, "Real-time protection of the JET ITER-like wall based on near infrared imaging diagnostic systems", 2018 Nucl. Fusion 58106021.

[4] ALVES, D. et al, "Vessel thermal map real-time system for the JET tokamak", 2012 Phys. Rev. ST Accel. Beams 15 054701 .

[5] HUBER, V. et al, "JUVIL: A new innovative software framework for data analysis of JET imaging systems intended for the study of plasma physics and machine operational safety”, 2017 Fusion Eng. Des. 123 979-985.

[6] HUBER, V. et al, 2018 submitted to Fusion Eng. Des.

[7] HUBER, A. et al 2018 submitted to Fusion Eng. Des.

[8] NIGHTINGALE, M. P. S. et al, “Overview on Experiments On ITER-like. Antenna On JET And ICRF Antenna Design”, 2009 AIP Conf. Proc. 1187, 213.

[9] WIDDOWSON, A. et al, "Overview of the JET ITER-like wall divertor", 2017 Nuclear Materials and Energy 12499 505.

[10] MAIER, H. et al, "Kinetics of carbide formation in the molybdenum-tungsten coatings used in the ITER-like Wall”, 2016 Phys. Scr. T167 014048.

[11] HERMANN, A., "Overview on stationary and transient divertor heat loads", 2002 Plasma Phys. Contr. Fusion 44883

[12] HUBER, V. et al, "In-vessel calibration of the imaging diagnostics for the real-time protection of the JET ITER-like wall”, 2016 Rev. Sci. Instrum. 87 11D430.

[13] BIEWER, T. M. et al, "Upgrades to the in-vessel calibration light source on JET”, 2012 Rev. Sci. Instrum. 8310 D505.

[14] NIT, New Imaging Technologies, http://new-imaging-technologies.com/en. 